\title{
How do voices become familiar? Speech intelligibility and voice recognition are differentially sensitive to voice training
}

\author{
Holmes, Emma ${ }^{1}$, To, Grace ${ }^{1}$, \& Johnsrude, Ingrid S. ${ }^{1,2}$ \\ ${ }^{1}$ The Brain and Mind Institute, University of Western Ontario, London, Ontario, N6A 3K7, \\ Canada \\ ${ }^{2}$ School of Communication Sciences and Disorders, University of Western Ontario, London, \\ Ontario, London, N6G 1H1, Canada
}

Corresponding author: Emma Holmes; E-mail: emma.holmes@ucl.ac.uk; Phone: +44 7597 967397; Mailing address: Wellcome Centre for Human Neuroimaging, University College London, 12 Queen Square, London, WC1N 3BG, United Kingdom. 
2 When people listen to speech in noisy places, they can understand more words spoken by 3 someone familiar, such as a friend or partner, than someone unfamiliar. Yet, we know little 4 about how voice familiarity develops. We exposed participants to three voices for different 5 lengths of time (speaking 88, 166, or 478 sentences). Trained voices were recognizable and 6 were more intelligible when presented with a competing talker than novel voices. However, 7 recognition and intelligibility improved at different rates with longer exposures, demonstrating 8 that recognition and intelligibility of familiar voices are partially dissociable. Whereas recognition 9 was similar for all trained voices, intelligibility was best for the most extensively trained voice. 10 The speech intelligibility benefit for the most extensively trained voice (10-15\%) is as large as 11 that reported for naturally highly familiar voices (friends and spouses) - demonstrating that 12 intelligibility of a voice can be improved substantially after only an hour of training. 


\section{Introduction}

We encounter familiar people every day. Most commonly, these are friends, partners, and family members-but we also encounter people who we know less well, such as work colleagues or television and radio presenters. As we get to know someone new, we develop the ability to recognize their identity from their voice. We are also better able to understand words spoken by familiar people than people we have never met (e.g., Domingo, Holmes, \& Johnsrude, 2020; Holmes, Domingo, \& Johnsrude, 2018; Johnsrude et al., 2013; Kreitewolf, Mathias, \& von Kriegstein, 2017; Levi, Winters, \& Pisoni, 2007; Nygaard \& Pisoni, 1998; Nygaard, Sommers, \& Pisoni, 1994; Yonan \& Sommers, 2000). However, our understanding of how people become familiar with new voices is relatively limited. Here, we investigated the extent to which recognition and intelligibility of a voice improve after brief training.

\section{Voice familiarity improves speech intelligibility}

Speech can be difficult to understand when several people speak at the same time ("cocktail party listening"; Cherry, 1953). Yet, when a competing talker is present, large intelligibility benefits have been demonstrated for voices that are highly familiar, such as a spouse the participant has been living with for more than 18 years (Johnsrude et al., 2013) or the participant's mother (Barker \& Newman, 2004). Even friends are substantially more intelligible than unfamiliar people (Domingo et al., 2020; Holmes et al., 2018). In fact, Domingo et al. (2020) found no significant difference in the magnitude of the intelligibility benefit for the voices of friends known for at least 1.5 years and the voices of long-term ( $>5$ years) spouses. People who had known each other for less than 1.5 years were not included in the study, raising the question —how much training on a voice is required to derive the asymptotic intelligibility benefit?

Several experiments have shown that the intelligibility of voices that have been trained in the lab over 2-9 days is better than that of unfamiliar voices (Kreitewolf et al., 2017; Nygaard \& Pisoni, 1998; Nygaard et al., 1994; Yonan \& Sommers, 2000). However, the magnitude of the speech intelligibility benefit seems to be smaller than for naturally familiar voices: Voice training improves word report by up to 10\% (Nygaard et al., 1994) or 0.52 decibels (Kreitewolf et al., 2017), whereas the benefit is $10-15 \%$ (Domingo et al., 2020; Domingo, Holmes, \& Johnsrude, 2019; Holmes et al., 2018) or 5-9 decibels (Domingo, Holmes, \& Johnsrude, 2019; Holmes \& Johnsrude, in press; Johnsrude et al., 2013) for friends' voices. These findings suggest that improved intelligibility of familiar voices is not all-or-none, but instead may depend on the length 
of exposure and/or the setting in which voices are encountered (trained or natural). Our first aim was to assess whether brief voice training could produce speech intelligibility benefits and, if so, how speech intelligibility relates to the length of time that participants have been trained on the voice.

\section{Recognition of familiar voices}

51 Participants are also able to explicitly recognize voices that have been trained in the lab (e.g., 52 Doddington, 1985; Nygaard \& Pisoni, 1998; Nygaard et al., 1994; Yonan \& Sommers, 2000).

53 Studies measuring speech intelligibility benefits from trained voices have typically measured 54 recognition of those voices at several times during training. For example, Nygaard and Pisoni 55 (1998) found that the ability to identify ten talkers improved steadily over nine days of training. 56 Yonan and Sommers (2000) trained participants by presenting 120 sentences spoken by 4 57 different talkers on two consecutive days; on each day of training, they were also tested on 58 voice identification for 80 sentences. Young adults' performance was almost perfect after only 59 one day of training.

60 It is unclear how new voices become recognizable following shorter exposures, and whether 61 improvements in speech intelligibility parallel improvements in recognition. The second aim of 62 this experiment was to compare explicit recognition of a voice to any speech intelligibility benefit 63 for the same voice. The acoustic features that are used to recognize voices and to derive the 64 intelligibility benefit from them are at least partially overlapping (Holmes et al., 2018; Nygaard \& 65 Pisoni, 1998; Nygaard et al., 1994; Remez, Fellowes, \& Rubin, 1997; Sheffert, Pisoni, Fellowes, 66 \& Ramez, 2012) However, Holmes et al. (2018) demonstrated that a familiar voice can benefit 67 intelligibility even if it is not explicitly recognizable. Thus, the intelligibility benefit from, and 68 recognition of, a trained voice may develop at different rates.

\section{Type of training}

70 Finally, given that the type of training has been proposed to influence voice learning (e.g., Case, 71 Seyfarth, \& Levi, 2018), our third aim was to compare two different training conditions: voices 72 presented alone and in the presence of multi-talker babble. In previous training studies, some 73 have trained in quiet (e.g., Nygaard \& Pisoni, 1998; Nygaard et al., 1994), whereas others have

74 trained in noise (e.g., Kreitewolf et al., 2017). However, no studies to date have directly

75 compared the speech intelligibility benefit for these different training conditions. 
Theoretically, we might expect the intelligibility benefit, and/or recognition, to differ when voices are trained in noise compared to in quiet. First, background noise may increase cognitive load during training (Mattys, Davis, Bradlow, \& Scott, 2012), making participants 'work harder' to recognize the voices. This idea is consistent with the finding that voices that are masked by background noise are better remembered (Hirshman, Trembath, \& Mulligan, 1994; Mulligan, 1996; Wayne \& Johnsrude, 2012). A second possibility is that noise guides listeners to learn parts of a voice that are most distinct from background noise (Mattys, White, \& Melhorn, 2005)—helping participants to better understand speech when it is masked by similar sounds.

84 This explanation is consistent with the theory of transfer appropriate processing (Morris,

85 Bransford, \& Franks, 1977), the encoding specificity hypothesis (Tulving \& Thomson, 1973), and with previous findings that the similarity of encoding and retrieval phases modulates the familiarvoice intelligibility benefit (Case et al., 2018). Alternatively, voices trained in quiet may be more intelligible, if background noise partially masks characteristics of a voice that are useful for recognition and intelligibility.

\section{Current experiment}

Here, we investigated how different amounts of experience with a voice affect recognition and intelligibility. We trained participants on three voices for different lengths of time-either 78, 156, or 468 sentences, corresponding to approximately 10,20, and 60 minutes of training. We then tested participant's ability to identify those voices and sentences spoken in them, in the presence of another talker. Half of the participants were trained on the voices with babble noise presented simultaneously, whereas the other half heard the voices alone (in quiet) during

97 training.

\section{Methods}

\section{Participants} We recruited 53 participants. Of these, 3 did not complete the experiment. The remaining 50 participants were age $18-28$ years $($ median $=18.6$ years, interquartile range $[I Q R]=1.1) ; 12$ participants were male, 36 were female, and 2 preferred not to disclose. A sample size of 50 provides 0.8 power to detect within-subject effects (i.e., among 4 familiarity conditions) of $f \geq$ 0.17 , between-subject effects (i.e., between 2 training groups) of $f \geq 0.32$, and within-between interactions of $f \geq 0.17$ (Faul, Erdfelder, Lang, \& Buchner, 2007). The familiar-voice benefit to speech intelligibility found in previous studies has a large effect size $[f=0.72$ in Johnsrude et al. 


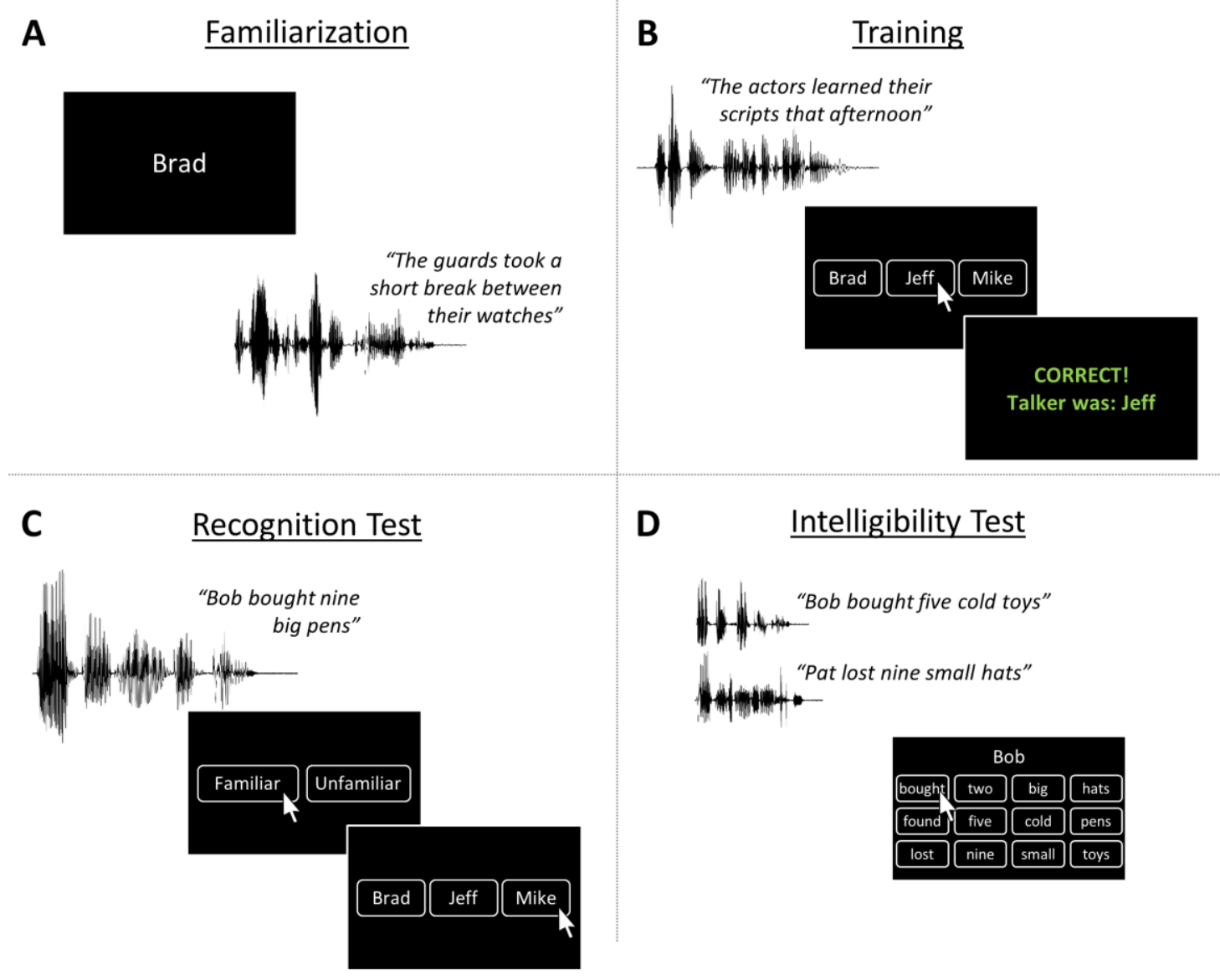

109 Figure 1. Schematics of the four phases of the experiment. (A) Familiarization: Participants saw 110 a name word on the screen (e.g. 'Brad') and heard a spoken sentence (e.g., 'The guards took a 111 short break between their watches'). (B) Training: Participants had to identify which of three 112 voices spoke each sentence. They received feedback about whether their response was correct 113 and were presented with the correct talker name. (C) Recognition test: Participants judged 114 whether sentences were spoken by familiar (trained) or unfamiliar (novel) talkers. If familiar, 115 they then had to select the name word that matched the voice. (D) Intelligibility test: Participants 116 heard two sentences, spoken simultaneously by different talkers. They had to attend to the 117 sentence that began with a particular name word (here, 'Bob'), and report the other four words 118 from the sentence by clicking one button from each column. Note only three rows of words are 119 shown here for clarity, but the experiment always contained eight rows, corresponding to all of 120 the words in the corpus. 
122 (2013) and $f=0.88$ in Holmes et al. (2018)], and familiarity effects of this size should be 123 detectable with power $\sim 1.00$ in the current design.

124 All participants were native Canadian English speakers and had no history of hearing difficulties.

125 They had average pure-tone hearing levels (measured at 5 octave frequencies between 0.5 and $1264 \mathrm{kHz}$ ) better than $15 \mathrm{~dB} \mathrm{HL}$ in each ear. The study was approved by Western University's

127 Health Sciences Research Ethics Board, and all participants gave informed consent.

\section{Design}

129 The experiment contained four parts: familiarization, training, an explicit recognition test, and a 130 speech intelligibility test. Schematics of the four tasks are displayed in Figure 1. Participants 131 completed all four parts in a single session, lasting approximately 4 hours.

132 Each participant heard three voices during the familiarization and training. During familiarization, 133 participants heard 10 sentences in each of the three voices (randomly interleaved). During 134 training, one of the voices was heard speaking 78 sentences, another 156 sentences, and the 135 third 468 sentences. In the explicit recognition and intelligibility tests, they heard the same three 136 voices and two other voices they had not previously heard. Across participants, the five talkers 137 were counterbalanced across familiarity conditions.

138 Half of participants $(\mathrm{N}=25)$ heard sentences alone during training (i.e., in quiet) and the other 139 half heard the training sentences in the presence of babble noise.

\section{Apparatus}

141 Acoustic stimuli were recorded using a Sennheiser e845-S microphone connected to a 142 Steinberg UR22 sound card (Steinberg Media Technologies) in a single-walled sound143 attenuating booth (Eckel Industries of Canada, Ltd.; Model CL-13 LP MR). Stimuli were 144 recorded in mono sound at a sampling rate of $44100 \mathrm{~Hz}$.

145 During the experiment, participants sat in a comfortable chair in the same single-walled sound146 attenuating booth facing a 24-inch LCD visual display unit (either ViewSonic VG2433SMH or 147 Dell G2410t). Acoustic stimuli were presented through the Steinberg UR22 sound card and 148 were delivered binaurally through Grado Labs SR225 headphones (Grado Labs, Brooklyn, 149 N.Y.). 
Table 1. Words from the Boston University Gerald (BUG) corpus used in this experiment

\begin{tabular}{lllll}
\hline Bob & bought & two & big & bags \\
\hline Pat & found & three & blue & cards \\
\hline & gave & four & cold & gloves \\
\hline held & five & hot & hats \\
\hline lost & six & new & pens \\
\hline & saw & eight & old & shoes \\
\hline & sold & nine & red & socks \\
\hline & took & ten & small & toys
\end{tabular}

\section{Stimuli}

155 Two different sentence corpora were used in this study: one for familiarization and training and

156 another for testing. For familiarization and training, we used meaningful sentences based on the 157 sentence corpus used by Rodd, Davis, and Johnsrude (2005), such as "The boy was able to

158 climb the mountain". We also created new sentences based on the syntactic and syllabic 159 structure of the existing sentences. We used 354 sentences in total (177 from Rodd et al., 160 2005), which are listed in Appendix 1.

161 For testing, we used closed-set sentences from the Boston University Gerald (BUG) corpus 162 (Kidd, Best, and Mason, 2008). These sentences each contain five words, in the form "Name 163 verb number adjective noun". An example is "Bob found three old socks". We used two Name 164 words ("Bob" and "Pat"). The other words each had eight possible options, which are displayed 165 in Table 1. We used a subset of 384 sentences. The probabilities of each pair of words 166 occurring together within a sentence were equated across the set.

167 We recorded five male talkers (age 20-24 years) speaking all 738 sentences. All talkers had a 168 Canadian accent and had no speech impediments. To ensure that all sentences were spoken at 169 similar rates, we played videos (Holmes, 2018) indicating the desired pace for each sentence 170 while participants completed the recordings. Participants saw the written sentence on the 171 screen, and were instructed to speak each word at the same time that a vertical bar passed the 172 beginning of the written word, but also to speak the sentences as naturally as possible. The 173 recorded familiarization and training sentences had an average duration of 3.1 seconds 174 (standard deviation [s.d.] = 0.7). The recorded test sentences had an average duration of 2.4 
175 seconds (s.d. = 0.2). The levels of recorded sentences were normalised for root mean square 176 (RMS) power.

177 The babble noise was a mixture of 12 (male and female) talkers, speaking different sentence 178 material, recorded in mono sound.

\section{Procedures}

180 Familiarization. During familiarization (Figure 1A), participants passively listened to 30 unique 181 high-context sentences. Of these, 10 sentences were spoken by each of three talkers. As 182 participants listened to each sentence, a name word-either Brad, Jeff, or Mike-was displayed 183 on the screen. Participants were asked to associate each of these names with the talker's voice. 184 After the sentence had ended, participants clicked with a computer mouse to hear the next 185 sentence. Across participants, the sentences spoken by each talker were counterbalanced, as 186 were the name words that were assigned to each voice. The familiarization procedure lasted 187 approximately 10 minutes.

188 Training. In the training phase (Figure 1B), participants completed 702 trials. Each trial 189 contained one sentence in one of the three familiarized voices. They heard one talker ("Most 190 Familiar") speak 468 sentences (i.e. 67\% of sentences), another talker ("Moderately Familiar") 191 speak 156 sentences (i.e., 22\% of sentences), and the remaining talker ("Least Familiar") speak 19278 sentences (i.e., 11\% of sentences). They heard 351 unique sentences during training, which 193 were each presented in two different voices: Across participants, the voices and sentences 194 assigned to the three conditions (Most Familiar, Moderately Familiar, and Least Familiar) were 195 counterbalanced,

196 Half of participants $(\mathrm{N}=25)$ heard the training sentences alone (i.e., in quiet), similar to the 197 familiarization, whereas the other half heard the same sentences in the presence of 198 simultaneous babble noise, presented at a signal-to-noise ratio (SNR) of $0 \mathrm{~dB}$. The babble noise 199 began 250 ms before the sentence began and ended 250 ms after the sentence had been 200 spoken.

201 After each spoken sentence had ended, a pop-up box appeared on the screen, prompting the 202 participant to indicate the name associated with the talker who spoke the sentence. Feedback 203 was provided after each response: text presented on the screen told participants whether they 204 had answered correctly or incorrectly, and displayed the correct name word; the text was 
coloured green if the participant had answered correctly and red if they had answered

206 incorrectly.

207 Training lasted approximately 1.5 hours, and was divided into 6 blocks, each containing 117 208 trials.

209 Explicit recognition test. The explicit recognition test was presented after training. It tested 210 whether participants recognized trained and novel voices as familiar and unfamiliar, and 211 whether participants could identify the names associated with the trained voices. During the 212 explicit recognition test (Figure 1C), participants heard BUG sentences spoken by the three 213 trained talkers and two novel talkers. After each sentence, they indicated whether they had 214 heard the talker during the training part or not. If they indicated they had heard the talker during 215 training, they were prompted to indicate the name of the talker. No feedback was provided. The 216 recognition task contained 105 sentences: 21 spoken by each of the five talkers.

217 Speech intelligibility test. Finally, participants completed a speech intelligibility test. On each trial 218 of the speech intelligibility test, participants heard two BUG sentences spoken by two different 219 talkers, which were presented simultaneously. The BUG sentences were different to those 220 presented during the explicit recognition test. The five words contained within the two 221 simultaneously presented sentences were always different. Participants were instructed to listen 222 to the target sentence that began with a specified name word ("Bob" or "Pat") and report the 223 remaining four words from that sentence by clicking words from a list of options on the screen, 224 in any order (see Figure 1D). Participants completed 640 trials; Each of the two Name words225 either "Bob" or "Pat" - was the target for half of trials (in separate blocks, in an order that was 226 counterbalanced across participants).

227 The target sentence could be spoken in any of the five voices, representing four training 228 conditions (Most Familiar, Moderately Familiar, Least Familiar, and Unfamiliar [two voices]). The 229 masker sentence was always in one of the two unfamiliar voices, and when the target was in an 230 unfamiliar voice, the other unfamiliar voice spoke the masker sentence. Equal numbers of trials 231 (160) were administered in the four conditions. Half of the masker sentences within each 232 condition were spoken by one of the novel talkers; the other half were spoken by the other novel 233 talker. This aspect of the design ensured that the masker talkers in all four conditions were 234 identical. Within each condition, we presented the sentences at two different target-to-masker 235 ratios (TMRs): -6 and $+3 \mathrm{~dB}$. 


\section{Analyses}

237 For the explicit recognition test, hits were defined as trials in which participants heard one of the 238 trained talkers and identified the correct name. Misses were defined as trials in which the 239 participant responded that the trained talker was unfamiliar, or an incorrect name was selected.

240 Hits and misses were calculated separately for the three trained talkers. Correct rejection and 241 false alarm rates were calculated from the 42 trials in which participants heard a novel voice. 242 Sensitivity (Hautus, 1995) was calculated for each of the three trained talkers. Chance $d^{\prime}$ was 2430.3 , and the maximum attainable $d^{\prime}$ was 4.3.

244 For the speech intelligibility test, we calculated the percentages of sentences that were reported 245 correctly for each of the 16 conditions (4 Familiarity conditions $\times 2$ TMR conditions $\times 2$ Training 246 Groups). We calculated the familiar-voice intelligibility benefit as the difference in percent 247 correct between the Unfamiliar baseline condition, and each of the three conditions in which a 248 trained talker was the target (Most Familiar, Moderately Familiar, and Least Familiar).

249 To examine whether the pattern of results across manipulations differed significantly between 250 the speech intelligibility and explicit recognition tasks, we converted d' from the explicit 251 recognition task and the percentage familiar-voice benefit to intelligibility into $z$-scores, which 252 were calculated separately for the two training groups.

253 Where Mauchley's Test of Sphericity was significant, we report statistics with Greenhouse 254 Geisser correction.

\section{Results}

\section{Training}

257 Recognition performance in the training conditions was high (Figure 2A). Performance averaged $25898.3 \%$ (S.E = 0.2) in the Most Familiar condition: 94.4\% (S.E = 1.0) in the Moderately Familiar 259 condition, and 94.3\% (S.E = 1.2) in the Least Familiar condition. A two-way mixed ANOVA 260 investigating whether performance during training differed across groups (quiet or babble; 261 between-subjects) and Familiarity conditions (Most Familiar, Moderately Familiar, Least

262 Familiar; within-subjects) revealed no effect of Group $\left[F(1,48)=0.36, p=0.55, \omega_{p}^{2}=-0.01\right]$ and 263 no significant interaction between Group and Familiarity $\left[F(2,96)=.21, p=.81, \omega_{p}^{2}=-0.02\right]$. 264 There was, however, a significant effect of Familiarity $\left[F(1.4,67.3)=12.64, p<0.001, \omega_{p}^{2}=\right.$ 265 0.19], with better performance in the Most Familiar condition than the Moderately Familiar 266 condition $\left[F(1,48)=18.04, p<0.001, \omega_{p}^{2}=0.25\right]$ and Least Familiar condition $[F(1,48)=12.50$, 
$\left.267 p=0.001, \omega_{p}^{2}=0.19\right]$. Performance did not differ between the Moderately Familiar and Least

268 Familiar conditions $\left[\mathrm{F}(1,48)=0.02, p=0.88, \omega_{p}^{2}=-0.02\right]$.

\section{Explicit recognition}

270 Percent correct in the Explicit Recognition test was good but below ceiling. The Most Familiar

271 voice was recognized correctly on $73.2 \%$ of trials, the Moderately Familiar voice was recognized

272 correctly on $73.1 \%$ of trials, and the Least Familiar voice was recognized correctly on $73.4 \%$ of

273 trials. The unfamiliar voices were correctly recognized as unfamiliar on $84.4 \%$ of trials.

274 Figure 2B shows the d'values in the Explicit Recognition test. We conducted a two-way mixed 275 ANOVA with the factors Training Group (quiet or babble) and Familiarity (Most Familiar, 276 Moderately Familiar, Least Familiar). Again, there was no effect of $\operatorname{Group}[\mathrm{F}(1,48)<0.01, p=$ $\left.2770.95, \omega_{p}^{2}=-0.02\right]$, and no interaction $\left[F(2,96)=2.58, p=0.08, \omega_{p}^{2}=0.03\right]$. There was also no 278 effect of Familiarity $\left[\mathrm{F}(2,96)=0.12, p=0.89, \omega_{p}^{2}=-0.02\right]$,

279

280

A

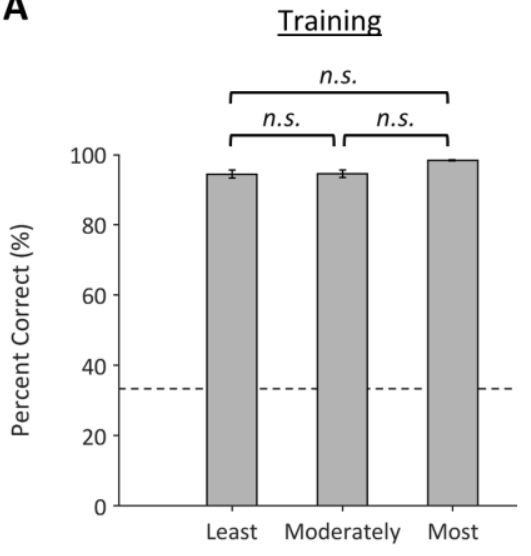

Talker Familiarity
B
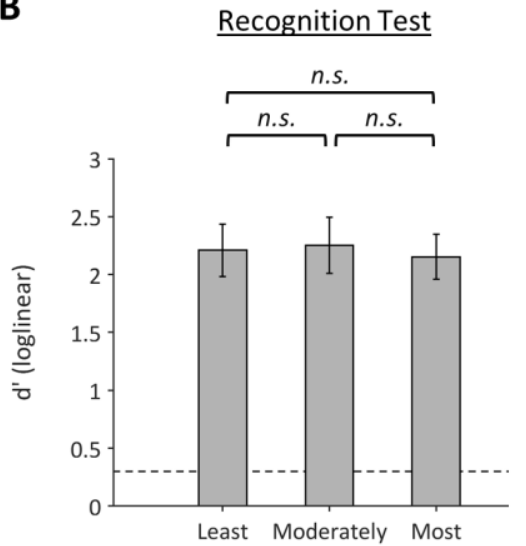

Talker Familiarity
C Intelligibility Test
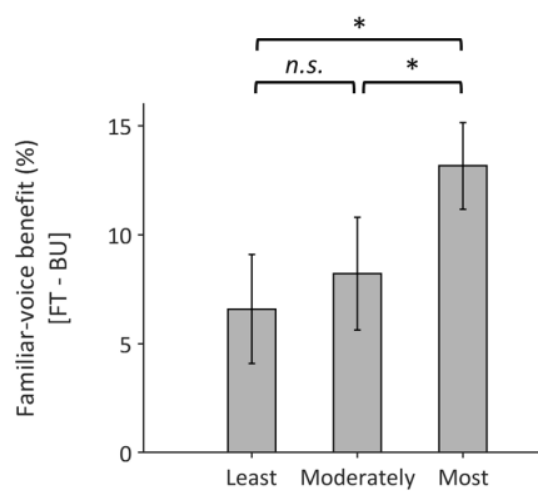

Talker Familiarity

Figure 2. Task performance by Familiarity. (A) Percent correct in the Training phase. The dashed line indicates chance performance (33\%). (B) d'values in the Explicit Recognition test.

284 The dashed line indicates chance d'. (C) The familiar-voice benefit to intelligibility, collapsed across target-to-masker ratios. The $y$-axis shows the difference in percent correct between each of the familiar conditions (FT) and the unfamiliar baseline (BU) condition. Error bars on all plots show \pm 1 standard error of the mean. Brackets show significance of pairwise comparisons $\left[{ }^{\star} p<\right.$ 0.05; n.s. not significant] 


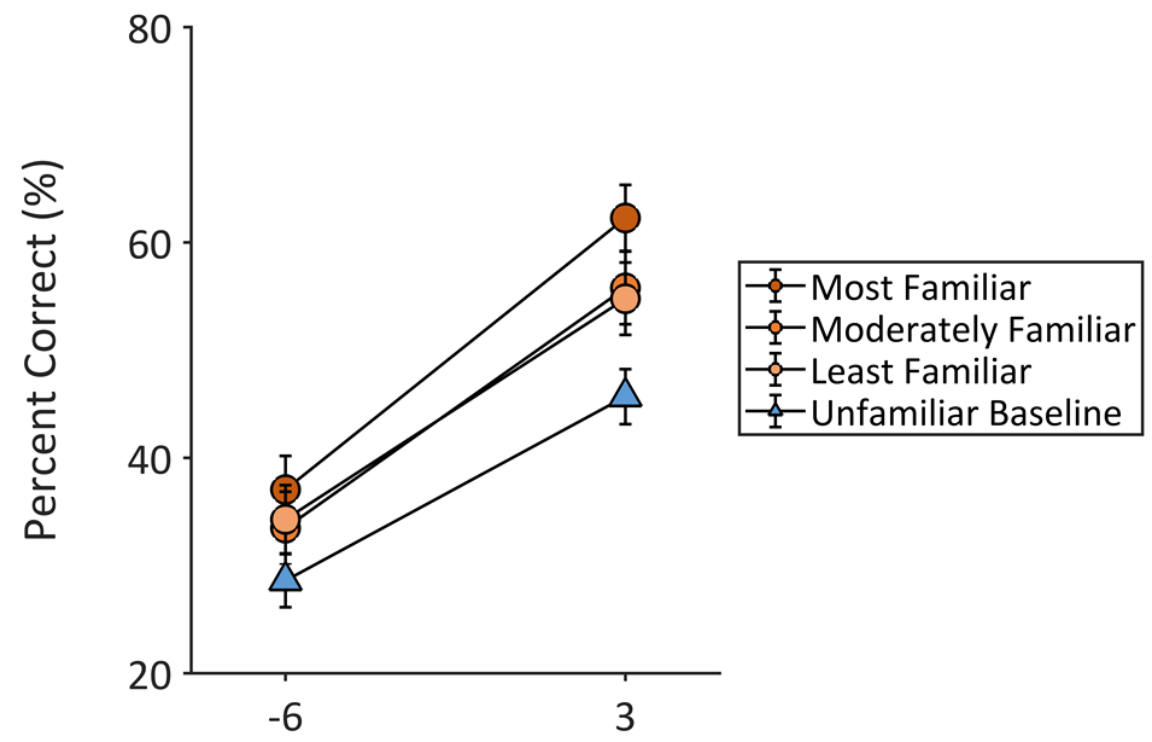

291 Figure 3. Results from the Speech Intelligibility Test in across Familiarity and Target-to-masker ratio (TMRs) conditions. Error bars show \pm 1 standard error of the mean.

295 Collapsing across Training Groups, we compared recognition d' in each Familiarity condition to 296 chance level $\left(d^{\prime}=0.3\right)$ using sign tests. Participants were able to identify all three voices with above-chance accuracy $(S \geq 40, p<0.001)$.

\section{Speech intelligibility}

299 Intelligibility data are shown in Figure 3. A three-way mixed ANOVA with the factors Training 300 Group (quiet or babble), Familiarity (Most Familiar, Moderately Familiar, Least Familiar, or 301 Unfamiliar), and TMR (-6 or $+3 \mathrm{~dB}$; within-subjects) revealed no effect of Group $[F(1,48)=0.20$, $302 p=0.66, \omega_{p}^{2}=-0.02$ ] and no interactions involving Group [Group x TMR: $F(1,48)=1.19, p=$ $3030.28, \omega_{p}^{2}<0.01$; Group x Familiarity: $F(3,144)=0.66, p=0.42, \omega_{p}^{2}=-0.01$; Group x Familiarity $304 \times$ TMR: $\left.F(3,144)=0.15, p=0.70, \omega_{p}^{2}=-0.02\right]$.

305 The effect of Familiarity was significant $\left[F(2.6,125.4)=10.49, p<0.001, \omega_{p}^{2}=0.16\right]$. Planned 306 contrasts showed that intelligibility was better in each of the three Familiar conditions than in the 
307 Unfamiliar condition $\left[F(1,48) \geq 6.81\right.$, ps $\left.<0.012, \omega_{p}^{2} \geq 0.10\right]$. Intelligibility was better in the Most

308 Familiar condition than in the Moderately and Least Familiar conditions [Moderately Familiar:

$309 F(1,48)=5.43, p=0.024, \omega_{p}^{2}=0.08$; Least Familiar: $F(1,48)=6.15, p=0.017, \omega_{p}^{2}=0.09$ ], but 310 the Moderately and Least Familiar conditions did not differ $\left[F(1,48)=0.52, p=0.48, \omega_{p}^{2}<-\right.$

$3110.01]$.

312 Intelligibility was significantly better at $+3 \mathrm{~dB}$ than $-6 \mathrm{~dB}$ TMR $\left[\mathrm{F}(1,48)=140.96, p<0.001, \omega_{p}^{2}\right.$

$313=0.74]$. There was an interaction between TMR and Familiarity $\left[F(3,144)=7.47, p<0.001, \omega_{p}^{2}\right.$

$314=0.12$ ], although the overall pattern across Familiarity conditions was preserved at the two

315 TMRs (see Figure 3); the interaction shows that TMR has a greater influence on intelligibility for 316 more familiar voices.

\section{Dissociation between recognition and intelligibility}

318 In the analyses above, there was a significant effect of Familiarity for speech intelligibility but not for explicit recognition. To examine whether the pattern of results across Familiarity Conditions differed between the speech-intelligibility and explicit-recognition tasks, we converted d' from the explicit-recognition task (Figure 2B) and the speech intelligibility benefit scores (Figure 2C) for each of the three familiar voices into $z$-scores and entered the data into a two-way withinsubjects ANOVA. Given that effects involving Training Group were never significant, we pooled the groups for this analysis. The two-way interaction between Task (explicit recognition or speech intelligibility) and Familiarity (Most, Moderately, or Least Familiar) was significant $[\mathrm{F}(1.8$, $\left.86.7)=4.55, p=0.017, \omega_{p}^{2}=0.07\right]$-confirming that the pattern of results across Familiarity conditions was different for the explicit-recognition and speech-intelligibility tasks.

\section{Discussion}

Our results demonstrate that recognition and improved speech intelligibility for trained voices emerge rapidly. Even for the Least Familiar talker, which participants were exposed to for approximately 10 minutes, we found successful voice recognition and a significant intelligibility 332 benefit.

333 We found a different pattern of results for explicit recognition and intelligibility, confirmed by a significant interaction between Task and Familiarity. Explicit recognition did not differ among the three voices that had been trained for different durations. However, speech intelligibility was best for the Most Familiar voice, and significantly lower for the Moderately and Least Familiar voices. Thus, whereas recognition is relatively stable over the range of exposures we tested, 
intelligibility is better after longer durations of training. These results contribute to an emerging idea that recognition and intelligibility of familiar voices are at least partially dissociable. A previous study (Holmes et al., 2018) provided evidence for this dissociation by showing that recognition and intelligibility of familiar voices are differentially sensitive to acoustic characteristics: some participants gained an intelligibility benefit for their friend's voice after it

343 had been manipulated in vocal tract length so it was not explicitly recognizable. In addition, a

344 functional magnetic resonance imaging (fMRI) study demonstrated distinct neural activation

345 when participants attended to the identity of a talker or the words they spoke (von Kriegstein, 346 Kleinschmidt, Sterzer, \& Giraud, 2005). Here, we provide new evidence for this dissociation by 347 showing that recognition and intelligibility of voices improve at different rates with training.

348 Recognition was uniformly high across talkers: as high as for the Least Familiar as for the Most 349 Familiar talker. These results cannot be explained by a ceiling effect: average d' was 2.2, whereas the maximum attainable $\mathrm{d}^{\prime}$ was 4.3 , and percent correct recognition was also below ceiling at $73 \%$. Thus, there was plenty of scope for recognition to differ between different talkers.

353 We found a significant intelligibility benefit for all three trained talkers, despite the fact that 354 training involved voice identification. This demonstrates that people learn aspects of familiar 355 voices that they rely on for improved intelligibility, even when training does not consist of an 356 intelligibility task.

357 Recognition and intelligibility were similar regardless of whether participants were trained to recognize the talkers in quiet or in simultaneously presented babble noise-reinforcing the idea that similar training and test conditions are unnecessary for familiar-voice learning. We measured speech intelligibility in the presence of a single competing talker, which is quite different to quiet and babble noise conditions. Our data provide no evidence for the encoding specificity hypothesis (Tulving \& Thomson, 1973), or for the idea that the additional challenge associated with listening in babble improves recognition or intelligibility—otherwise, we should have found better intelligibility and recognition for participants who were trained in babble than those who were trained in quiet. These findings demonstrate that the benefit to intelligibility is relatively robust and is not overly sensitive to voice training conditions.

367 The benefit that we observed for the Most Familiar talker (10-15\%) is similar in magnitude to 368 that previously reported for naturally familiar voices with the same task (Domingo et al., 2020; 369 Holmes \& Johnsrude, in press). This highlights the great potential of voice training for improving 
370 intelligibility in everyday settings. Voice training may be particularly beneficial for older people and/or people with hearing loss who experience particular difficulty listening in noisy settings

372 (e.g., Dubno, Dirks, \& Morgan, 1984). There is already some evidence that older people (Yonan 373 \& Sommers, 2000)—and older people with confirmed hearing loss (Souza, Gehani, Wright, \& 374 McCloy, 2013)—benefit from familiar-voice information. Unlike previous experiments that tested 375 intelligibility of trained talkers after two or more days of training (Kreitewolf et al., 2017; Nygaard 376 \& Pisoni, 1998; Nygaard et al., 1994; Yonan \& Sommers, 2000), our Most Familiar talker was 377 trained for only about one hour. This duration of training is comparable to everyday situations in 378 which people become familiar with new colleagues, or with radio and television presenters. The 379 short duration of the training used here highlights that voice training could be an accessible way 380 to improve speech intelligibility in everyday settings. Such benefits may also be relevant for occupations that require accurate speech perception when other sounds are present; for example, aircraft pilots.

In summary, we show that relatively little ( 10 minutes) training is sufficient for listeners to identify someone's identity from their voice and to realize a benefit to intelligibility when a competing talker is present. Nevertheless, we found that recognition and intelligibility develop over different time scales. Recognition was similar for three talkers that were trained for different lengths of time ( 10-60 minutes), but intelligibility was best for the talker that had received the most ( $\sim 1$ hour of) training. Overall, our results demonstrate the great potential of voice training for improving intelligibility in everyday settings, and also show a dissociation between intelligibility and recognition of trained voices.

\section{Acknowledgements}

This work was supported by funding from the Canadian Institutes of Health Research (CIHR; Operating Grant: MOP 133450) and the Natural Sciences and Engineering Research Council of 394 Canada (NSERC; Discovery Grant: 327429-2012). We would like to thank Brian Gygi for 395 sharing the babble sound file that we used for this experiment.

\section{References} infant streaming. Cognition, 94, 45-53. https://doi.org/10.1016/j.cognition.2004.06.001

Case, J., Seyfarth, S., \& Levi, S. V. (2018). Short-term implicit voice-learning leads to a Familiar Talker Advantage: The role of encoding specificity. The Journal of the Acoustical Society of America, 144(6), EL497-EL502. https://doi.org/10.1121/1.5081469 
402

403

404

405

406

407

408

409

410

411

412

413

414

415

416

417

418

419

420

421

422

423

424

425

426

427

428

429

430

431

432

433

434

435

436

437

438

439

440

441

442

443

Cherry, E. C. (1953). Some experiments on the recognition of speech, with one and two ears. The Journal of the Acoustical Society of America, 25(5), 975-979.

Doddington, G. R. (1985). Speaker Recognition-Identifying People by their Voices. Proceedings of the IEEE, 73(11), 1651-1664. https://doi.org/10.1109/PROC.1985.13345

Domingo, Y., Holmes, E., \& Johnsrude, I. S. (2020). The benefit to speech intelligibility of hearing a familiar voice. Journal of Experimental Psychology: Applied. Advance online publication. https://doi.org/10.1037/xap0000247

Domingo, Y., Holmes, E., Macpherson, E., \& Johnsrude, I. S. (2019). Using spatial release from masking to estimate the magnitude of the familiar-voice intelligibility benefit. The Journal of the Acoustical Society of America, 146(5), 3487-3494.

Dubno, J. R., Dirks, D. D., \& Morgan, D. E. (1984). Effects of age and mild hearing loss on speech recognition in noise. The Journal of the Acoustical Society of America, 76(1), 8796. Retrieved from http://asadl.org/jasa/resource/1/jasman/v76/i1/p87_s1

Faul, F., Erdfelder, E., Lang, A.-G., \& Buchner, A. (2007). G*Power 3: A flexible statistical power analysis program for the social, behavioral, and biomedical sciences. Behavior Research Methods, 39(2), 175-191.

Hautus, M. J. (1995). Corrections for extreme proportions and their biasing effects on estimated values of d'. Behavior Research Methods, Instruments, \& Computers, 27(1), 46-51. https://doi.org/10.3758/BF03203619

Hirshman, E., Trembath, D., \& Mulligan, N. (1994). Theoretical implications of the mnemonic benefits of perceptual interference. Journal of Experimental Psychology: Learning, Memory, and Cognition, 20(3), 608-620. https://doi.org/10.1037/0278-7393.20.3.608

Holmes, E., Domingo, Y., \& Johnsrude, I. S. (2018). Familiar voices are more intelligible, even if they are not recognized as familiar. Psychological Science, 29(10), 1575-1583. https://doi.org/10.1177/0956797618779083

Holmes, E., \& Johnsrude, I. S. (in press). Speech spoken by familiar people is more resistant to interference by linguistically similar speech. Journal of Experimental Psychology: Learning, Memory, and Cognition. Retrieved from https://doi.org/10.31234/osf.io/2ebrs

Johnsrude, I. S., Mackey, A., Hakyemez, H., Alexander, E., Trang, H. P., \& Carlyon, R. P. (2013). Swinging at a cocktail party: voice familiarity aids speech perception in the presence of a competing voice. Psychological Science, 24(10), 1995-2004. https://doi.org/10.1177/0956797613482467

Kreitewolf, J., Mathias, S. R., \& von Kriegstein, K. (2017). Implicit talker training improves comprehension of auditory speech in noise. Frontiers in Psychology, 8, 1584. https://doi.org/10.3389/fpsyg.2017.01584

Levi, S. V, Winters, S. J., \& Pisoni, D. B. (2007). A Cross-Language Familiar Talker Advantage? (Vol. 28).

Mattys, S. L., Davis, M. H., Bradlow, A. R., \& Scott, S. K. (2012). Speech recognition in adverse conditions: A review. Language and Cognitive Processes, 27(7-8), 953-978. https://doi.org/10.1080/01690965.2012.705006

Mattys, S. L., White, L., \& Melhorn, J. F. (2005). Integration of multiple speech segmentation cues: A hierarchical framework. Journal of Experimental Psychology: General, 134(4), 
Morris, C. D., Bransford, J. D., \& Franks, J. (1977). Levels of processing versus transfer appropriate processing. Journal of Verbal Learning and Verbal Behavior, 16, 519-533.

Mulligan, N. W. (1996). The effects of perceptual interference at encoding on implicit memory, explicit memory, and memory for source. Journal of Experimental Psychology: Learning, Memory, and Cognition, 22(5), 1067-1087. https://doi.org/10.1037/0278-7393.22.5.1067

Nygaard, L. C., \& Pisoni, D. B. (1998). Talker-specific learning in speech perception. Perception \& Psychophysics, 60(3), 355-376. https://doi.org/10.3758/BF03206860

Nygaard, L. C., Sommers, M. S., \& Pisoni, D. B. (1994). Speech perception as a talkercontingent process. Psychological Science, 5(I), 42-46.

Remez, R. E., Fellowes, J. M., \& Rubin, P. E. (1997). Talker identification based on phonetic information. Journal of Experimental Psychology: Human Perception and Performance, 23(3), 651-666. https://doi.org/10.1037/0096-1523.23.3.651

Sheffert, S. M., Pisoni, D. B., Fellowes, J. M., \& Ramez, R. E. (2012). Learning to recognise talkers from natural, sinewave, and reversed speech samples. J Exp Psychol Hum Percept Perform, 28(6), 1447-1469. https://doi.org/10.1016/j.biotechadv.2011.08.021.Secreted

Souza, P. E., Gehani, N., Wright, R., \& McCloy, D. (2013). The advantage of knowing the talker. Journal of the American Academy of Audiology, 24(January 2013), 689-700. https://doi.org/10.3766/jaaa.24.8.6

Tulving, E., \& Thomson, D. M. (1973). Encoding specificity and retrieval processes in episodic memory. Psychological Review, 80(5), 352-373. https://doi.org/10.1037/h0020071

von Kriegstein, K., Kleinschmidt, A., Sterzer, P., \& Giraud, A.-L. (2005). Interaction of face and voice areas during speaker recognition. Journal of Cognitive Neuroscience, 17(3), 367376. https://doi.org/10.1162/0898929053279577

Wayne, R. V, \& Johnsrude, I. S. (2012). The role of visual speech information in supporting perceptual learning of degraded speech. Journal of Experimental Psychology: Applied, 18(4), 419-435. https://doi.org/10.1037/a0031042

Yonan, C. A., \& Sommers, M. S. (2000). The effects of talker familiarity on spoken word identification in younger and older listeners. Psychology and Aging, 15(I), 88-99. https://doi.org/10.1037/0882-7974.15.1.88 